\title{
Contingencies of Self-Worth and Social-Networking-Site Behavior
}

\author{
Michael A. Stefanone, Ph.D., ${ }^{1}$ Derek Lackaff, Ph.D., ${ }^{2}$ and Devan Rosen, Ph.D. ${ }^{3}$
}

\begin{abstract}
Social-networking sites like Facebook enable people to share a range of personal information with expansive groups of "friends." With the growing popularity of media sharing online, many questions remain regarding antecedent conditions for this behavior. Contingencies of self-worth afford a more nuanced approach to variable traits that affect self-esteem, and may help explain online behavior. A total of 311 participants completed an online survey measuring such contingencies and typical behaviors on Facebook. First, exploratory factor analyses revealed an underlying structure to the seven dimensions of self-worth. Public-based contingencies explained online photo sharing $(\beta=0.158, p<0.01)$, while private-based contingencies demonstrated a negative relationship with time online $(\beta=-0.186, p<0.001)$. Finally, the appearance contingency for self-worth had the strongest relationship with the intensity of online photo sharing $(\beta=0.242)$, although no relationship was evident for time spent managing profiles.
\end{abstract}

\section{Introduction}

$\mathbf{M}$ EDIA SHARING HAS EMERGED as one of the preeminent online activities of the "social web." Over half of all Internet-using teens are "content creators" who create Web sites or blogs, share original media like photos and videos, or remix content into new creations. ${ }^{1}$ Social-networking sites like MySpace (http://myspace.com) and Facebook (http:// facebook.com) are becoming cornerstones of this informational space, with many recent surveys finding that $95 \%$ or more of college students maintain site profiles. ${ }^{2}$ Facebook reports that its users upload over 2 billion photos per month and 14 million videos per week, in addition to billions of other pieces of content such as blog posts and web links, ${ }^{3}$ and research is beginning to focus on antecedents and consequences of such behavior.

Online social media represent significant new opportunities for involvement in broader media cultures. Celebrities and everyday people alike use microblogging platforms like Twitter to communicate with "followers" and "fans," and provide personal photos to be commented upon and discussed by friends and strangers. An evolving culture of transparency and disclosure has been noted and often lamented by scholars of traditional mass media, ${ }^{4}$ yet the use of online platforms for intimate self-expression has become a key component of the overall social environment for many.
Previous studies have found relationships between traditional mass media and new media behaviors, ${ }^{5}$ suggesting that a cultural fascination with celebrity may contribute to the ways new communication platforms are appropriated.

This study focuses on a particular type of media-sharing behavior, the sharing of personal photos, as well as several other types of ordinary social-network site (SNS) behavior. As cameras have become ubiquitous and ever present (primarily as they have been integrated with mobile communication devices), photography has become more than an archival process. Photos do not just commemorate important events and special occasions, but record our everyday lives and social interactions. Unlike the textual media that has formed the basis of online communication for most of its existence, personal photos are intrinsically intimate, even as the ubiquity of Photoshop promotes skepticism about the truthfulness of images. Counts and Fellheimer ${ }^{6}$ suggest that photo sharing serves to enhance the social presence of individuals to their close friends and family, and helps establish a common social milieu among groups. However, when presented in public or semipublic fora such as a Flickr album or Facebook profile, photos may signal the existence of relationships, a desire for relationships, or even a desire for attention.

Because of the questions surrounding the motivations associated with sharing pictures online, this paper situates photo-sharing behavior within a more personal context.

${ }^{1}$ Department of Communication, University at Buffalo, Buffalo, New York.

${ }^{2}$ Department of Radio, Television and Film, University of Texas, Austin, Texas.

${ }^{3}$ Department of Speech, University of Hawaii, Manoa, Hawaii. 
Specifically, we examine self-esteem (SE) and contingencies of self-worth $(\mathrm{CSW})^{7,8}$ as antecedents for the sharing of personal photos and behaviors such as friending (profile linking), developing large online networks, and spending time managing the profile's appearance and data. We further suggest that gender plays an important role in the ways in which identitymediating technologies are utilized. Users' overall (offline) social environments are also taken into account in our analyses. We first present a brief review of the literature on SE and motivations for media sharing. This background is followed by a discussion of the research design and the results, which suggest that CSW contribute in different ways to online behavior. Conclusions are offered along with recommendations for future research.

\section{Antecedents of Media-Sharing Behavior}

Digital devices have radically transformed the social landscape of photography. Digital cameras began to outsell film cameras in 2004, ${ }^{9}$ while camera-enabled mobile phones began to outsell digital cameras just a year later. ${ }^{10}$ The profusion of digital cameras and camera devices suggest that digital photography-like other new media practices-is an increasingly banalized activity ${ }^{11}$ that may play a subtle but important role in social relationships. A host of web services such as Flickr and Snapfish (http://snapfish.com) have emerged to support the storage, organization, and sharing of digital photos, while general social-networking sites like Facebook and MySpace include photo sharing as a key functionality. For example, users upload in excess of 2.5 billion photos to Facebook each month. ${ }^{3}$

Photography has a long and social history. As Americans have grown increasingly mobile in recent decades, photos have helped keep distant family members and friends in close emotional proximity. In many cases, the digitalization of photography simply made these sharing processes faster and more convenient. The web has also enabled relatively new forms of interaction through photos. Miller and Edwards ${ }^{12}$ note that two relatively distinct modes of photo sharing can be observed online- both the traditional sharing of photos with an existing social network of friends and family, and an emergent form of public sharing with strangers and online acquaintances. These two groups are perhaps better understood as representing ends of a spectrum of sharing behaviors, as the boundaries of intimacy are increasingly blurred by technological affordances.

Some previous studies have examined media sharing and attempted to provide explanations for user behavior. Stefanone et al. ${ }^{13}$ found that many types of online behavior exhibited by social web users are related to the consumption of particular types of "old media," particularly "reality television." They argued that users attempt to produce and maintain their online identity within an overall media context. Those users whose personal media context is dominated by reality television were found to exhibit higher levels of behaviors, including time devoted to social-network profile maintenance, size of the online network, promiscuous friending (creating online profile links to people not met in person), and photo sharing. These findings were presented within the framework of social cognitive theory, ${ }^{14}$ with the suggestion that these online behaviors may reflect identification with fame-seeking and competitive behaviors observed in reality television.
Stefanone and Lackaff ${ }^{5}$ examined blogging, photo, and video-sharing behaviors online as forms of non-directed selfdisclosure. In addition to measuring the consumption of television content, this study took into account authoritarianism, a personality variable. The authors hypothesized that authoritarianism would serve to enhance the behavioral impacts of television consumption, but this hypothesis was not supported by their analysis. Two demographic factors-age (younger) and sex (female)—were significant predictors of posting photographs online. Younger people posted more photos, and females were more likely to post photos than males. The female image maintains strong connotations in our culture, and this finding may indicate that young females are following broader cultural prescripts about image use. In addition to personality variables, this study also attempted to situate behavior in a social context. Here, the size of participants' social-support networks demonstrated a significant positive relationship with photo-posting behavior. People who had more close friends and family shared more pictures online.

While this model explained photo-sharing behavior, their results suggest that other factors contribute to this behavior. They suggested that posting photos can contribute to relationship maintenance and may also promote reciprocal behavior. The high numbers of photos shared by some participants (many hundreds, in some cases) points towards the value that they attribute to sharing personal images. This "personal value" might be attributed to self-presentation goals, ${ }^{15}$ which are themselves a function of an individual's SE.

\section{Contingencies of Self-worth and Achievement Motivation}

SE refers to one's appraisal of the value or worth of the self. While SE has been a common measure in social psychology, recent research shows that different people look to different domains of their life in their SE appraisals, necessitating multidimensional approaches to SE measurement. The CSW model presents one such framework.7,8

Crocker and Wolfe ${ }^{7}$ found that existing understanding of SE tended to be too simplistic, and that the relationship between low SE and social problems had been overemphasized. In particular, they argue that behavior is better and more reliably understood as a function of domain-based, rather than global, forms of SE. Such CSW develop over an individual's life in response to multiple forms of socialization, thus they are relatively stable but not immutable.

Seven domains of contingency have been identified by Crocker et al., 16 including competencies, competition, approval from generalized others, family support, appearance, God's love, and virtue. Briefly, competencies refer to specific abilities like academic competence; competition refers to outdoing others; approval from generalized others refers to the perception of others' esteem; family support refers to perceived affection and love from family members; appearance refers to self-evaluations of one's physical appearance; God's love refers to the belief that one is valued by a supreme being; and virtue refers to adherence to a moral code. ${ }^{16}$

Importantly, Crocker et al. ${ }^{16}$ found that different CSW actually predicted different types of behavior as measured by time spent participating in different activities, and the contingency effects were significant even when controlling for 
generalized SE. The appearance CSW, for example, predicted partying/socializing, shopping, joining social groups such as fraternities/sororities, and grooming, but had a negative relationship with spiritual activities and time spent with family. Further, the (academic) competency CSW demonstrated a positive relationship with time studying and a negative relationship with partying.

In general, people will enact behaviors that enhance their self-worth within their particular contingent domains. The patterns observed by Crocker et al. ${ }^{16}$ confirm that CSW have a self-regulation function, influencing the domains in which people direct their energies. One expression of selfworth-related behavior is manifest via identity construction and maintenance.

\section{Self-presentation goals and contingencies of self-worth}

CSW regulate behavior because SE is tied to success within specific contingencies. Regardless of high or low overall SE levels, people seek positive feelings associated with success in their CSW and avoid negative feelings associated with failure in these domains. Further research suggests that CSW are related to goals because success within CSW feeds selfvalidation goals. ${ }^{17}$ If people are unsure about their chances for success in these domains, or if failure can be avoided, they will stop engaging in that behavior. The self-worth theory of achievement motivation suggests people are motivated to construct an image of themselves as competent. ${ }^{18}$ This CSW is based on competence and is an example of a self-preservation goal. Crocker and Knight suggest "the importance of SE lies less in whether it is high or low, and more in what people believe they need to be or do to have value and worth as a person."19(p200)

Crocker and Park ${ }^{17}$ argue that the pursuit of SE can be costly. Although they cite short-term benefits like increased happiness and decreased anxiety, these boosts do not satisfy fundamental needs for learning, relatedness, and autonomy. They further suggest that the boosts to SE within relevant domains (CSW) can be addicting, and there are real costs to pursuing esteem by validating successes. For example, in terms of costs to relationships, the pursuit of SE conflicts with the initiation and maintenance of mutually supportive relationships, as people become focused on themselves, rather than the needs and feelings of others. ${ }^{17}$ In summary, they argue that relationships in fact become a tool to validate the self, as opposed to an opportunity to give to and support others in equitable, reciprocal relationships.

\section{Social media and identity management}

One space where the strategic manipulation of relational and social information is readily apparent is online communication platforms. Online spaces such as social-network sites and media-sharing sites can be understood to heighten people's consciousness of the ways in which their identities are socially constructed (for example ${ }^{15}$ ). Rather than simply being the target of mediated messages, they can see themselves as protagonists of mediated narratives who actively integrate themselves into a complex media ecosystem. The media tools and strategies traditionally employed by celebrities and their handlers-airbrushed photos, carefully coordinated social interactions, strategic selection, and maintenance of the entourage-are now in a sense available to everyone with a computer, and are increasingly employed in everyday interpersonal interaction. Today, much online communication is thus marked by an increasing emphasis on existing offline relationships, physical and nonverbal communication cues, and their manipulation.

There are also documented gender differences in communication technology use. Weiser ${ }^{20}$ discovered that female Internet users tend to seek health and education information online, whereas males prefer searching for news and entertainment purposes. In terms of social uses of technology, Boneva and Kraut $^{21}$ suggested that women are more likely to use online tools to maintain or extend their social networks than their counterparts. These differences may result from different attitudes toward relationships. Tannen ${ }^{22}$ suggested that females have specific communication goals, including fostering community and avoiding isolation, while males are more likely to pursue social positioning through communication. For example, women use the telephone more often than men for relational goals like keeping in touch with distant friends. ${ }^{23}$ Ramirez and Broneck ${ }^{24}$ also found that females are more likely to use instant messaging for maintaining relationships than males. Rosen et al. ${ }^{25}$ found that women share more photos on social-networking sites and spend more time maintaining those sites. This evidence suggests female students spend more time on mediated communication than male students for relationship maintenance, and suggests systematic differences in terms of social-network composition.

In social-network analysis, the most frequently measured aspect of interpersonal relationships is tie strength, or intensity, an indication of how close a respondent reports being to each network member. ${ }^{26}$ Strong-tie contacts are characterized by frequent, reciprocal communication and usually a long, stable history of interaction. Often, strong ties constitute relationships with family and close friends. In contrast, weak ties are characterized by infrequent communication, low reciprocity, and a lack of emotional closeness. ${ }^{27,28}$ Some individuals have large egocentric networks comprised of numerous weak-tie contacts, while others have smaller egocentric networks with few strong ties.

The proportions of weak and strong ties in ego networks are often a function of the time necessary to maintain those relationships. However, strong-tie relationships are more costly to maintain, given their richness and intensity. As the quantity of these costly relationships increases in a given social network, so should the motivation to use cost-reducing technologies like social-network sites to communicate with these close friends and family members. In this way, communication and photo sharing through these Web sites can serve to maintain existing relationships.

These network and relationship principals and genderrelated findings have significant implications for the understanding of online photo-sharing behavior. As reviewed above, photo sharing has a long and rich history, and functions to support relationships. Further, females are more likely to communicate with relationship maintenance goals. Thus the following hypotheses are based on gender differences: H1. Female participants have larger offline social support
networks than male participants.

H2. Female participants (a) spend more time managing online profiles, (b) share more photos online, (c) have larger mediated social networks, and (d) tend to friend people they actually 
have interpersonal relationships with, compared to male participants.

However, there is also the possibility that females identify more strongly with their image and appearance, and use Facebook as a platform to compete for attention. If this is the case, then females should be more likely to engage in promiscuous friending online whereby they make formal online friend connections with people they have not actually met face to face. As such, we propose this competing hypothesis to $\mathrm{H} 2 \mathrm{c}$ above:

H3. Female participants are more likely to friend others never actually met face to face, as opposed to males.

Given the body of research suggesting that the social context of technology use is an important explanatory variable regarding use of contemporary communication technology, ${ }^{29-32}$ this study affords the opportunity to explore the influence of people's offline, traditional social networks. Therefore, we propose the following hypothesis:

H4. Offline social support network size has a positive relationship with (a) time spent managing social-network site profiles, (b) online photo sharing, and (c) online network size.

However, it is likely that people need some (minimum) level of self-confidence or SE to engage in this behavior. People base their esteem on different contingencies, ${ }^{16}$ and these contingencies likely contribute to explanations of some online behaviors. As reviewed above, Park ${ }^{31,32}$ offers CSW as a more nuanced conceptual framework from which to evaluate esteem and behavior.

On the one hand, social-network sites may function as tools through which people maintain meaningful relationships with people they have known for a long time and are emotionally close to (see $\mathrm{H} 2 \mathrm{~b}$, above). On the other hand, networking sites may serve as a platform to compete for attention. Recall that the shift from people consuming online content to producing it was paralleled by a cultural shift toward visibility, transparency, and celebrity. ${ }^{5}$ In this regard, attention can be operationalized as a basis for social power, and people compete in imperfect markets for this scarce resource. One attention-seeking strategy might be to share photos of oneself. This behavior has the added benefit of affording people a high level of control over their self-presentation.

Given the range and diversity of CSW available in the literature and the relative novelty of mass online photo-sharing behavior, the current study adopts an exploratory approach to investigating these relationships. Thus the following research question is proposed:

RQ1: What is the relationship between the range of CSW and (a) time spent managing online profiles, (b) online photo sharing, and (c) online friending behavior?

However, one CSW likely has a clear relationship with behavior on social-networking sites. Appearance-based CSW should have a positive relationship with both investing resources into managing online profiles and online photo sharing. Therefore, the following hypothesis is proposed:

H5. Appearance-based CSW has a positive relationship with (a) time spent managing online profiles and (b) online photo sharing.

\section{Method}

Pilot study

Because some of the variables used in this study were single-item measures, a pilot study was conducted prior to the main data collection for this study. The purpose of the pilot study was to test the validity of single-item measures in terms of the accuracy of respondent recall. Two items were selected to test in the pilot study: the size of online social networks and the number of photographs shared. These items were chosen because exact, objective measurement of these variables is easily accessible by simply viewing online profiles. We acknowledge that single-item measures have the potential for attenuation. However, when measures are in reference to precise, singular objects like the number of friends or the number of photos shared online, there is evidence that single items have comparable reliability and predictive validity as multiple-item scales. ${ }^{33,34}$

A total of 100 students volunteered from an undergraduate class and were given research credit for participating. Of these, 53 participants were female, and the entire group averaged 19.5 years of age $(S D=1.87)$. For the recall data, participants reported an average of 259 network contacts $(S D=202)$ and shared an average of 84.4 photos $(S D=73.8)$. Pearson correlation coefficients between the recall and actual data were 0.64 and 0.61 for network size and number of photos shared respectively.

To determine if systematic differences existed in recall based on age and gender, and to test how well recall data predicted actual data, two separate regression models were calculated. The model predicting network size was significant, $F(3,100)=33.15, p<0.0001$; the recall variable was the only significant predictor $(\beta=0.61, p<0.0001)$ of actual network size. Similarly, the regression model for number of photos shared was significant, $F(3,100)=29.05, p<0.0001$, and the recall data was the only significant predictor $(\beta=0.59, p<0.0001)$. These results suggest that young people are able to recall the size of their online networks and the number of photos shared with a fair amount of accuracy, and no systematic differences were apparent in terms of age or gender.

\section{Main study}

A total of 311 online surveys were completed by a sample of university students. Students were recruited from introductory communication classes and granted research credit for their participation, in accordance with Institutional Review Board protocols for student research involvement. Approximately $49.8 \%$ of the sample was female; the average age of participants was 23.3 years $(S D=3.47)$. These participants did not significantly differ from pilot-study participants in terms of demographic composition. The majority of participants identified their ethnic background as Caucasian (approximately 64\%). About 14\% were Asian, 6\% were African-American, and 3\% were Hispanic. The rest (about $13 \%$ ) identified with a variety of other ethnicities.

Offline strong-tie networks. Because people have the capacity to identify accurately people they have frequent interaction with, ${ }^{35}$ offline (or traditional) strong-tie network size was measured using a single item that specifically explained the detailed uniqueness of strong-tie affiliations by focusing on relationship duration, communication frequency, 
and emotional closeness. On average, participants reported having $7.65(S D=4.68)$ close friends and family members.

Social-network site measures. To measure the length of time spent logged into social-network sites, participants were asked two questions assessing how many hours and minutes they spend online. Participants reported spending an average of $56.2(S D=39.7)$ minutes per session and maintained network profiles for an average of $2.58(S D=1.38)$ years. This is consistent with average Facebook user statistics. ${ }^{3}$ In general, two users are either "friends," or they have no relationship whatsoever. This particular constraint is articulated by socialnetwork platforms, and one question was used to measure how many "friends" participants are linked to via their network sites. Participants reported an average of 267.33 friends $(S D=222.05)$.

Because this study is interested in whether people use networking sites to maintain meaningful relationships or to compete for attention, an additional measure was used to estimate the proportion of online networks that reflect actual interpersonal relationships. Participants were asked to report the number of friends in their online network who they never actually met face to face. On average, participants reported not having met $12 \%$ of their online social networks $(S D=0.21)$. This variable was skewed and log transformed to normalize $(M=1.14, S D=0.73)$. These results are consistent with previous research by Stefanone et al., ${ }^{13}$ and suggest that people are somewhat promiscuous in terms of their online friending behavior.

Finally, participants were asked to indicate the number of photographs of themselves they have publicly available on their network site profiles $(M=115.34, S D=196.79)$. The number of Facebook friends and photos shared online were both heavily skewed and were log transformed for analyses $(M=0.45, S D=0.47$ and $M=1.60, S D=0.82$ respectively). Means, standard deviations, and Cronbach's alphas are included in Table 1.

CSW measures. CSW were measured using the scale items developed by Crocker and Wolfe. ${ }^{7}$ These items were used to measure all seven contingencies: approval, appearance, competition, academic achievement, family support, virtue, and God's love (Likert scales where $7=$ "strongly agree"). The descriptive statistics and Cronbach's alphas for each measure are summarized in Table 1.

It is evident from the results that many of the individual scales in the CSW measures correlated with each other. Based on exploratory factor analysis $(\mathrm{KMO}=0.718$, Bartlett's Test: $\left.\chi^{2}=241.63, p<0.001\right)$ using principal components extraction with varimax rotation, a two-factor solution explaining $56.6 \%$ variance was found (see Table 2). Examination of the scree plot confirmed the two-factor solution. Academic achievement cross-loaded on both factors and was withdrawn from further consideration in this study. The first factor was comprised of elements related to more traditional, personal domains like family, virtue, and God's love. We label this factor private sphere contingencies $(\alpha=0.72)$. The second factor focused on approval, appearance and competition concepts, and we label this factor public sphere contingencies $(\alpha=0.69)$. These public-sphere contingencies relate more to public interaction and evaluation, and may be more likely associated with online sharing of personal media like photographs.

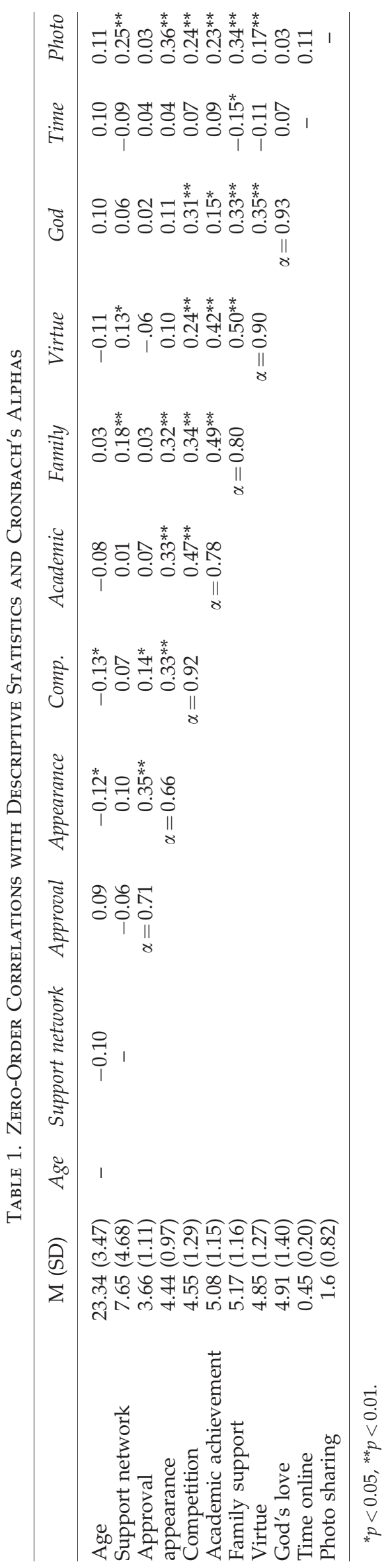


TAble 2. Component Matrix for Factor ANALYSIS OF CSW Items

\begin{tabular}{lll}
\hline & \multicolumn{2}{c}{ Component } \\
\hline & 1 & 2 \\
Approval & & 0.741 \\
Appearance & & 0.827 \\
Competition & & 0.545 \\
Academic achievement & 0.520 & 0.511 \\
Family support & 0.721 & \\
Virtue & 0.810 & \\
God's love & 0.657 & \\
\hline
\end{tabular}

Average responses for the new public- and private-sphere scales were $5.02(S D=1.36)$ and $4.21(S D=1.20)$ respectively.

\section{Results}

As a validity check for the two-factor structure of CSW, separate ordinary least squares (OLS) regression models were used to test the relationship between the private and public factors, and offline strong-tie networks. If these factors accurately measure two different dimensions of CSW, then private CSW should have a positive relationship with the tendency to develop meaningful, emotionally close relationships regardless of technology; the public CSW should not. Results show that the public-factor CSW did not have a significant relationship with offline strong-tie network size. However, the private factor was a significant predictor $(\beta=0.136, p<0.01)$ of the number of strong ties participants had, $F(3,305)=4.51, p<0.01$. These results lend support to the validity of the underlying factor structure revealed in the factor analyses.

Correlations reveal that younger participants reported being more focused on appearance and competition CSW, while those with larger social support networks identified more closely with family and virtue-related CSW. Strong-tie network size also had a positive relationship with online photo sharing. None of the CSW had a significant relationship with either time spent managing Facebook profiles or the frequency of online photo sharing.

A one-way analysis of variance (ANOVA) with gender as the between-group variable was used to test whether differences in strong-tie network size existed. Female participants had an average of $8.2(S D=5.2)$ close friends and family, while males had $7.1(S D=3.9)$ strong ties. This difference was significant, $F=3.69, p<0.05$, support for hypothesis 1 . Additional ANOVAs were calculated to explore genderbased differences in CSW. Results show that females reported significantly stronger associations with appearance $(p<0.001)$, academic $(p<0.001)$, family support $(p<0.001)$, and virtue $(p<0.05)$ CSW. Gender-based differences were also apparent for the two-factor solution (public, private CSW) described above. Female participants more strongly identified with both dimensions $(p<0.001)$. Further, male and female participants both reported significantly stronger identification with the private CSW factor ( $t$ test, $p<0.01$ ), opposed to the public factor. This may be indicative of a systematic bias in the form of a social desirability response effect.

Ordinary least squares regression was used to test the other hypothesized relationships in this study. The first analyses summarized in Table 3 were used to test hypotheses 2, 3, and 4. Age, gender, strong-tie network, and public-sphere CSW were regressed onto time spent managing networking-site profiles in Model I. This model was significant, F (5, $224)=2.92, p<0.05$, and explained $4 \%$ of the total variance. Public CSW were not a significant predictor, but gender was. Female participants spent more time managing their online profiles $(\beta=-0.176, p<0.01)$, giving support for hypothesis 2a. In Model II, the same set of independent variables was regressed onto the online photo-sharing variable. This model was also significant, $F(4,247)=11.98, p<0.01$, and explained over $15 \%$ of the total variance. Public CSW was significant in this model $(\beta=0.158, p<0.01)$. Participants who identified with public sphere-based CSW shared more photos online, as did female participants, providing support for hypotheses $2 \mathrm{~b}$.

The same independent variables were regressed onto the Facebook (online) network size in Model III and the promiscuous friending variable in Model IV. Gender did not explain online network size or promiscuous friending; hypotheses $2 \mathrm{c}$ and $2 \mathrm{~d}$ were not supported. Although female participants were trending toward promiscuity online $(\beta=-0.130, p=0.07)$, this relationship was not significant; hypothesis 3 was not supported either. In Model IV, younger participants had larger online networks $(\beta=0.199, p<0.001)$ and were more promiscuous in their friending $(\beta=0.238$, $p<0.001$ ). However, because none of the hypotheses was supported, these results were excluded from Table 3.

Hypothesis 4 stated that offline strong-tie networks have a positive relationship with time spent maintaining online socialnetwork profiles, online network size, and online photo

Table 3. Public-Based CSW Regressed onto Time-Managing Profiles (Model I, F[5, 224]=2.92,* Adj. $R^{2}=0.041$ ), Online Photo Sharing (Model II, $F[4,247]=11.98$, ${ }^{* *}$ Adj. $R^{2}=0.151$ ), and Online Network Size (Model III, $F[5,224]=3.97,{ }^{* *}$ AdJ. $R^{2}=0.05$ )

\begin{tabular}{|c|c|c|c|c|c|c|}
\hline & \multicolumn{2}{|c|}{ Model I (time) } & \multicolumn{2}{|c|}{ Model II (photo) } & \multicolumn{2}{|c|}{ Model III (online network size) } \\
\hline & SE & $\beta$ & SE & $\beta$ & SE & $\beta$ \\
\hline Age & 0.015 & 0.089 & 0.022 & 0.102 & 0.016 & $0.199^{* *}$ \\
\hline Gender & 0.062 & $-0.176^{*}$ & 0.098 & $-0.234^{*}$ & 0.065 & 0.092 \\
\hline STN & 0.006 & -0.113 & 0.100 & $0.222^{*}$ & 0.007 & $0.147^{*}$ \\
\hline Public CSW & 0.037 & -0.020 & 0.060 & $0.158^{*}$ & 0.039 & 0.012 \\
\hline
\end{tabular}

${ }^{*} p<0.05 ;{ }^{* *} p<0.01$. 
sharing. Referring to Model I in Table 3, it is clear that strong-tie network size did not have a positive relationship with time spent managing profiles. However, Model II shows that there was a significant relationship between strong ties and online photo sharing $(\beta=0.222, p<0.01)$ and online network size $(\beta=0.147, p<0.05)$. Although hypothesis $4 \mathrm{a}$ was not supported, hypotheses $4 \mathrm{~b}$ and c were.

Next, private-sphere CSW were regressed onto the same two dependent variables to complete the comparative analyses and to address the research question proposed in this study. Model I in Table 4 explaining time spent managing profiles was significant, $F(4,224)=4.97, p<0.001$, and explained about $6.6 \%$ of the variance. Once again, female participants spent the most time managing profiles. Model II was significant as well, $F(4,247)=12.14, p<0.001$, and explained more than $15 \%$ of the total variance. Interestingly, private CSW exhibited a negative relationship with time spent managing profiles $(\beta=-0.186, p<0.001)$; participants who identified strongly with family, values, and spirituality spent less time online. However, private CSW had the opposite relationship with online photo sharing $(\beta=0.165$, $p<0.01)$. Female participants, those who identified with the private CSW factor, and those with larger social support networks invested more heavily in online photo sharing. These results support hypotheses $4 \mathrm{~b}$ and c. Hypothesis $4 \mathrm{a}$ was not supported.

The last set of analyses is summarized in Table 5. These models were used to explore the relative and combined contributions of appearance and private-sphere CSW on time spent managing SNS profiles and online photo sharing. To test hypothesis 5a, which stated a positive relationship between appearance CSW and time spent managing profiles, the first regression analysis included only appearancebased CSW as an independent variable. This model was not significant; hypothesis 5a was not supported. Model I in Table 5 includes both appearance and private-sphere CSW. The addition of appearance CSW weakened the relationship between private sphere CSW and time spent managing profiles, but appearance CSW did not explain time online. Model II explaining photo sharing was significant, $F(4$, $248)=14.62, p<0.001$, and explained over $18 \%$ of the variance. In this model, appearance-based CSW was a strong predictor, $\beta=0.242, p<0.001$, of online photo sharing, thereby supporting hypothesis $5 \mathrm{~b}$. Note that the beta is stronger than all other betas presented in these analyses. Model III incorporated both appearance CSW and the private CSW scale and was significant, $F(5,247)=12.84$, $p<0.001$, and explained almost $20 \%$ of the total variance.
Although strong-tie network size and gender were significant in both models, Model III explained the most variance by including both the private-sphere CSW scale and the specific appearance-based CSW.

\section{Discussion}

The purpose of this study was to investigate variables that function as antecedents to specific online behaviors. This behavior was operationalized along a range of online activity germane to social-network sites like Facebook and included time spent managing their profiles, the size of their online networks, how promiscuous they were in terms of their friending behavior, and online photo sharing. Given the popularity of this set of online behavior, the results of this study make a timely contribution to the body of research directed at people's behavior on popular social-networking sites. One of the strengths of this research lies in the design, whereby participant's offline social context was incorporated into the analyses.

We began by incorporating Crocker et al.'s ${ }^{16}$ and Park' ${ }^{32}$ recent scholarship on SE and CSW. After reviewing this literature, it was apparent that a number of the specific contingencies could add explanatory power to models of people's online behavior beyond traditional measures of traitbased SE. However, correlation analyses revealed that many of the contingencies were related. Therefore, exploratory factor analysis was used and revealed a satisfactory twofactor solution. Appearance, approval, and competitionbased contingencies were classified as public contingencies through which people base their self-worth. These were deemed public in the sense that everyday interaction with other people is the basis for these comparisons and evaluations. Appearance, for example, highlights the importance of other people's evaluations of how one looks. Of course, one's appearance is "public" in the sense that there are few restrictions on who can access this information. Similarly, the desire for approval from others is deemed public because the satisfaction of approval needs is contingent upon interaction with others.

Private contingencies are different because they address more traditional, personal, and spiritual elements. Take family support, for example. This form of social support is derived from long-term emotionally close relationships. Among relationships like these, the content of communication is characteristically much more intimate and personal. In effect, one's boundaries of privacy tend to be much more relaxed in these situations ${ }^{36}$ and social exchange reflects this.

Table 4. Private-Based CSW Regressed onto Time-Managing Profiles (Model I: F[4, 224] $=4.97$, $^{* * *}$ Adj. $R^{2}=0.066$ ), Online Photo Sharing (Model II, $F[4,247]=12.14{ }^{* * *}$ Adj. $R^{2}=0.157$ ), And Online Network Size (Model III, F[4, 229] $=4.47,{ }^{* *}$ AdJ. $\left.R^{2}=0.057\right)$

\begin{tabular}{|c|c|c|c|c|c|c|}
\hline & \multicolumn{2}{|c|}{ Model I (time) } & \multicolumn{2}{|c|}{ Model II (photo) } & \multicolumn{2}{|c|}{ Model III (online network size) } \\
\hline & SE & $\beta$ & SE & $\beta$ & SE & $\beta$ \\
\hline Age & 0.015 & 0.097 & 0.022 & $-0.117^{*}$ & 0.026 & $0.218^{* * * *}$ \\
\hline Gender & 0.06 & $-0.200^{* *}$ & 0.097 & $-0.234^{* * *}$ & 0.029 & 0.095 \\
\hline STN & 0.006 & -0.086 & 0.01 & $0.198^{* *}$ & 0.030 & $0.140^{*}$ \\
\hline Private CSW & 0.031 & $-0.186^{* * *}$ & 0.048 & $0.165^{* *}$ & 0.015 & 0.092 \\
\hline
\end{tabular}

${ }^{*} p<0.05 ;{ }^{* *} p<0.01 ;{ }^{* * *} p<0.001$. 
Table 5. Added Contribution of Appearance CSW to Online Photo Sharing

\begin{tabular}{|c|c|c|c|c|c|c|}
\hline & \multicolumn{2}{|c|}{ Model I (time) } & \multicolumn{2}{|c|}{ Model II (photo) } & \multicolumn{2}{|c|}{ Model III (photo) } \\
\hline & SE & $\beta$ & SE & $\beta$ & SE & $\beta$ \\
\hline Age & 0.015 & 0.085 & 0.021 & 0.108 & 0.022 & 0.110 \\
\hline Gender & 0.062 & $-0.219^{* *}$ & 0.101 & $-0.189^{* *}$ & 0.099 & $-0.175^{* *}$ \\
\hline STN & 0.006 & -0.093 & 0.011 & $0.217^{* * *}$ & 0.014 & $0.196^{* *}$ \\
\hline Appearance CSW & 0.032 & 0.025 & 0.075 & $0.242^{* * *}$ & 0.051 & $0.221^{* * *}$ \\
\hline Private CSW & 0.033 & $-0.176^{*}$ & - & - & 0.048 & $0.130^{*}$ \\
\hline
\end{tabular}

Model I: $F(X, 247) 3.65,{ }^{* *}$ Adj. $R^{2}=0.056$; Model II: $F(4,247)=14.62{ }^{* * *}$ Adj. $R^{2}=0.181$; Model III: $F(5,247)=12.84$, Adj. $R^{2}=0.193$. ${ }^{*} p<0.05 ;{ }^{* *} p<0.01 ;{ }^{* * *} p<0.001$.

There is congruence between this connection and the private nature of ideals regarding perceptions of virtuous behavior and individual spirituality as well.

It is important to note that these two factors do not operate independently of each other, nor are they mutually exclusive. Rather, the goal of this research was to explore the relative contributions of each set of contingencies on people's behavior online. As much of this research was exploratory in nature, future research should start to focus systematically on individual contingences, similar to how the appearance CSW was examined in this study.

The first set of hypotheses addressed gender differences in on- and offline behavior. Female participants reported having significantly larger networks of strong ties, as expected. Considering the different motivations for communication and relationships, this finding regarding offline behavior is not surprising. In fact, strong-tie network size was an influential variable in many of the analyses presented herein. For example, participants with larger networks shared more photos online. Strong, intimate relationships are generally more costly than weak-tie relationships in terms of time and effort to maintain. People who have many such relationships in their lives may be more likely to utilize technological affordances that cheaply convey positive affect (see for exam$\mathrm{ple}^{32}$ ). Although the variable was not addressed in this study, it seems likely that people post photos not only of themselves but of themselves in the company of others. Such photos may serve to communicate the importance of particular relationships because these bonds may provide security regarding ones appearance and self-worth.

Park $^{31}$ notes that anxiety over expectations of being rejected or accepted by others based on appearance is inherently interpersonal. As such, studies investigating appearance-based CSW should begin to include behaviors in interpersonal relationships along varying intensity from early stage relationships where people may be increasingly attentive to appearance to close relationships that may provide a safeguard to appearance threats. It is along these lines that future research should attempt to tease out these nuances of self-presentation regarding photo sharing in social media, as the outcome of these instrumental decisions may provide users with tools to mitigate uncertainty surrounding their perceived appearance.

There were systematic gender differences in behavior throughout the results of this study. Females tended to spend more time managing their profiles and shared more photos online. This was the case regardless of which dimension of self-worth influenced their behavior, and re- gardless of the size of their offline social support network. Females also identified more strongly with the appearance CSW. Such findings may suggest persistent differences in gendered behavior that result from a cultural focus on female image and appearance. Further, the culture of celebrity alluded to in the introduction offers significantly more appearance-based rewards and penalties to women than to men.

It is interesting to note that the private-sphere CSW factor explained more variance than the public-sphere CSW. We suspected that a focus on contingencies that look to more public aspects of personal life and identity would result in more participation in these online behaviors. For example, people who base their self-worth on others' evaluations of their appearance should be more likely to emphasize their appearance in online interactions by sharing more photos of themselves. However, it may also be the case that people who base their SE on appearance do not necessarily believe that they are capable of competing socially in this domain. Clearly, one can be dissatisfied with one's appearance, yet still believe that appearance is an important component of one's self-worth. Positive and negative CSW in the publicsphere domain may tend to average out, reducing their impact in our analyses. Those who base their self-worth on private-sphere CSW may be less affected by this variance.

One area for future research to explore is the negative relationship between private CSW and time spent managing profiles, and the opposite relationship observed between private CSW and online photo sharing. It seems these people use social media to share images of themselves with their strong-tie networks but are less interested in actively managing their online persona. However, the nature of this relationship remains unclear.

Finally, CSW did generally explain behavior in expected ways, but there are several reasons for the range of explanatory power in the models presented in the results section. First, people engage in activities and behaviors for a multitude of reasons. For example, people may share a large number of photos because their self-worth is at stake, or they may simply be interested in photography and use these tools to showcase their work. Second, there are many ways to satisfy specific CSW, several motivations can result in the same outcome, and several outcomes can be related to the same contingency. ${ }^{16}$ While CSW did add to the explanatory models presented herein, the nuances of these psychological concepts need to be refined in more controlled laboratory settings.

In conclusion, contemporary online communication tools facilitate low-cost distribution of personal media and infor- 
mation. This manuscript argues that individual traits are antecedent to much of the social behavior enacted online. Contingencies on which people assess their self-worth represent a new approach to understanding how personal identities are developed and maintained. This study provides a framework for future explorations of identity construction, social interaction, and media use in a rapidly changing communication environment.

\section{Disclosure Statement}

No competing financial interests exist.

\section{References}

1. Lenhart A, Madden M. Teen content creators and consumers. Washington, DC: Pew Internet \& American Life Project 2005. www.pewinternet.org/PPF/r/166/report_display .asp (accessed Mar. 20, 2009).

2. Profile of the American College Student (PACS) Survey. (2007) Profile of the American College Student: University of Missouri-Columbia. Columbia, MO: Institutional Research, UMC. //ir.missouri.edu/reports-presentations.html (accessed Mar. 20, 2009).

3. Facebook. (2010) www.facebook.com/press/info.php? statistics (accessed Mar. 20, 2009).

4. Calvert C. (2000) Voyeur nation: Media, privacy and peering in modern culture. Boulder, CO: Westview Press.

5. Stefanone MA, Lackaff D. Reality television as a model for online behavior: Blogging, photo and video sharing. Journal of Computer-Mediated Communication 2009; 14: 964-87.

6. Counts S, Fellheimer E. (2004) Supporting social presence through lightweight photo sharing on and off the desktop. In Proceedings of the SIGCHI Conference on Human Factors in Computing Systems. Vienna, Austria, pp. 599-606.

7. Crocker J, Wolfe CT. Contingencies of self-worth. Psychological Review 2001; 108:593-623.

8. Wolfe CT, Crocker LE. (2002) What does the self want? A contingencies of self-worth perspective on motivation. In Kunda Z, Spencer S, eds. The Ontario Symposium: Goals and motivated cognition. Hillsdale, NJ: Erlbaum, pp. 147-170.

9. Musgrove, M. Nikon says it's leaving film-camera business. Washington Post, Jan. 12, 2006, D01. www.washingtonpost. com/wpdyn/content/article/2006/01/11/AR2006011102323. html (accessed Oct. 22, 2007).

10. Sharma DC. Study: Cameraphone market will top digital cameras. CNET News.com, Aug. 10, 2005. www.news.com/ Study-Camera-phone-market-will-top-digital-cameras /21001041_3-5827024.html (accessed Oct. 22, 2007).

11. Graham S. Beyond the "dazzling light": from dreams of transcendence to the "remediation" of urban life. New Media \& Society 2004; 6:33-42.

12. Miller AD, Edwards WK. (2007) Give and take: a study of consumer photo-sharing culture and practice. In: Proceedings of the SIGCHI Conference on Human Factors in Computing Systems. San Jose, CA: ACM, pp. 347-56.

13. Stefanone MA, Lackaff D, Rosen D. (2008) We're all stars now: Reality television, Web 2.0, and mediated identities. In Proceedings of ACM's Hypertext and Hypermedia. Los Alamitos, CA: IEEE Press, pp. 107-12.

14. Bandura A. (1986) Social foundations of thought and action: A social cognitive theory. Englewood Cliffs, NJ: Prentice-Hall.

15. Toma CL, Hancock JT, Ellison N. Separating fact from fiction: An examination of deceptive self-presentation in online dating profiles. Personality \& Social Psychology Bulletin 2008; 34:1023-36.

16. Crocker J, Luhtanen RK, Cooper ML, et al. Contingencies of self-worth in college students: Theory and measurement. Journal of Personality \& Social Psychology 2003; 85:894-908.

17. Crocker J, Park L. The costly pursuit of self-esteem. Psychological Bulletin 2004; 130:392-414.

18. Covington MV. Goal theory, motivation, and school achievement: An integrative review. Annual Review of Psychology 2000; 51:171-200.

19. Crocker J, Knight KM. Contingencies of self-worth. Current Directions in Psychological Science 2005; 14:200-3.

20. Weiser E. Gender differences in Internet use patterns and Internet application preferences: A two-sample comparison. CyberPsychology \& Behavior 2000; 3:167-77.

21. Boneva B, Kraut R. (2002) Email, gender, and personal relationships. In Wellman B, Haythornthwaite C, eds. The Internet in everyday life. Malden, MA: Blackwell, pp. 520-48.

22. Tannen D. (1992) You just don't understand: Women and men in conversation. London: Virago Press.

23. Lacohee, $\mathrm{H}$, Anderson B. Interacting with the telephone. Journal of Human-Computer Studies 2001; 54:665-99.

24. Ramirez A, Broneck K. IM me: Instant messaging as relational maintenance and everyday communication. Journal of Social \& Personal Relationships 2009; 26:291-315.

25. Rosen D, Stefanone MA, Lackaff D. (2010) Online and offline social networks: Investigating culturally-specific behavior and satisfaction. In Proceedings of IEEE's Hawaii International Conference on Systems Science. Los Alamitos, CA: IEEE Press.

26. McCarty $\mathrm{C}$. The meaning of knowing as a network tie. Connections 1996; 18:20-31.

27. Granovetter M. The strength of weak ties. American Journal of Sociology 1973; 78:1360-79.

28. Granovetter M. (1983) The strength of weak ties: A network theory revisited. In Marsden P, Lin N, eds. Social structure and network analysis. Beverly Hills: Sage, pp. 105-30.

29. Franzen A. Does the Internet make us lonely? European Sociological Review 2000; 16:427-38.

30. Stafford L, Kline SL, Dimmick J. Home e-mail: Relational maintenance and gratification opportunities. Journal of Broadcasting \& Electronic Media 1999; 43:659-69.

31. Park LE. Appearance-based rejection sensitivity: Implications for mental and physical health, affect, and motivation. Personality \& Social Psychology Bulletin 2007; 33:490-504.

32. Park LE, Crocker J. Contingencies of self-worth and responses to negative interpersonal feedback. Self \& Identity 2008; 7:184-203.

33. Wanous JP, Hudy MJ. Single-item reliability: A replication and extension. Organizational Research Methods 2001; 4:361-75.

34. Bergkvist L, Rossiter JR. The predictive validity of multipleitem versus single-item measures of the same constructs. Journal of Marketing Research 2007; 44:175-84.

35. Marsden PV. Network data and measurement. Annual Review of Sociology 1990; 16:435-63.

36. Petronio SS. (2002) The boundaries of privacy: Dialectics of disclosure. Albany, NY: State University of New York Press.

Address correspondence to: Dr. Michael A. Stefanone

Department of Communication

University at Buffalo

359 Baldy Hall

Buffalo, NY 14260

E-mail: ms297@buffalo.edu 
